\title{
High density H-mode operation by pellet injection and ELM mitigation with the new active in-vessel saddle coils in ASDEX Upgrade
}

P. T. Lang, W. Suttrop, E. Belonohy, M. Bernert, R.M. Mc Dermott, R. Fischer, J. Hobirk, O.J.W.F. Kardaun, G. Kocsis 1), B. Kurzan, M. Maraschek, P. de Marne, A. Mlynek, P.A. Schneider, J. Schweinzer, J. Stober, T. Szepesi 1), K. Thomsen 2), W. Treutterer, E. Wolfrum, ASDEX Upgrade Team

MPI für Plasmaphysik, EURATOM Association., 85748 Garching, Germany

1) KFKI RMKI, EURATOM Association, P.O.Box 49, H-1525 Budapest-114, Hungary

2) EFDA Close Support Unit Garching, Boltzmannstrasse 2, 85748 Garching, Germany

E-mail contact of main author: peter.lang@aug.ipp.mpg.de

Short tile: High density ELM mitigated H-mode in AUG

PACS numbers: 52.35.Py, 52.55.Fa, 52.55.Tn

\begin{abstract}
Recent experiments at ASDEX Upgrade demonstrate the compatibility of ELM mitigation by magnetic perturbations with efficient particle fuelling by inboard pellet injection. ELM mitigation persists in a high density, high collisionality regime even with the strongest applied pellet perturbations. Pellets injected into mitigation phases trigger no type-I ELM like events unlike when launched into unmitigated type-I ELMy plasmas. Furthermore, the absence of ELMs results in improved fuelling efficiency and persistent density build up. Pellet injection is helpful to access the ELM-mitigation regime by raising the edge density beyond the required threshold level, mostly eliminating the need for strong gas puff. Finally, strong pellet fuelling can be applied to access high densities beyond the density limit encountered with pure gas puffing. Core densities of up to 1.6 times the Greenwald density have been reached while maintaining ELM mitigation. No upper density limit for the ELM-mitigated regime has been encountered so far; limitations were set solely by technical restrictions of the pellet launcher. Reliable and reproducible operation at line averaged densities from 0.75 up to 1.5 times the Greenwald density is demonstrated using pellets. However, in this density range there is no indication of the positive confinement dependence on density implied by the ITERH98P(y,2) scaling.
\end{abstract}




\section{Introduction}

The power load deposited by type-I ELMs onto the first wall and divertor presents a severe danger for ITER. Currently, there are several options for ELM mitigation: Operating with plasma scenarios which develop no or only benign ELMs, ELM pace-making to enhance the ELM frequency by at least a factor of $15-30$ in order to reduce individual ELM losses by this same factor, or ELM mitigation or ELM suppression with non-axisymmetric magnetic perturbations. Pace-making tries to reduce the type-I ELM size by triggering the instability through an external perturbation, e.g. pellet injection with a rate higher than the natural ELM frequency. Avoidance of type-I ELMs is preferred over ELM pace-making if it can be achieved without significant deleterious impact on the plasma performance. Nonaxisymmetric magnetic perturbations have been investigated in DIII-D where full suppression or at least significant mitigation of ELMs has been achieved over a wide operational range [1]. This successful first demonstration prompted further experiments at JET [2,3], NSTX [4] and MAST [5] with different perturbation field configurations. In theses experiments, quite different results with respect to ELM mitigation were obtained, and so far full ELM suppression has not been reproduced in any of them. Recently, the ASDEX Upgrade tokamak has been enhanced with a set of in-vessel coils [6] in order to study ELM amelioration, shed light on the physics involved, and to improve the extrapolation base for ITER. In a first stage of this enhancement, $n=2$ magnetic perturbations are found to allow suppressing type-I ELMs and replace them with very benign ELM activity [7].

A key issue for H-mode operation with ELM suppression or mitigated ELM activity is whether core pellet fuelling, a requirement in ITER, will trigger strong ELMs and thereby reintroduce excessive peak power loads. In DIII-D pellets were injected during phases with type-I ELM suppression achieved by resonant magnetic perturbation (RMP) in order to counteract the density pump out caused by coil activation. Density increase was enforced by pellets but at the expense of a transient return of type-I ELMs [8]. Pellet fuelling was also applied at JET for a fully successful recovery of the density reduction caused by the actuated external coils. Here RMP resulted in a reduction of the ELM size with respect to encountered energy loss and divertor power flux by a factor of about 4 with the ELM frequency increased accordingly. Every injected fuelling size pellet triggered a type-I ELM, adding a few extra type-I ELMs very similar in size to the spontaneous ELMs, which remained during the mitigation phase [9].

Both the DIII-D and JET observations are in line with experimental observations of the ELM triggering potential of pellets in different regimes at ASDEX Upgrade [10]. Pellet-triggered ELMs show, in some cases with minor modifications, the same behaviour as spontaneous ELMs typical for the corresponding scenario. This behaviour was interpreted as being due to the strong pellet pressure perturbation in the edge providing the stimulus for a pressure-driven mode, which triggers an ELM that would otherwise have evolved later spontaneously. This qualitative picture has been essentially confirmed by modelling employing the non-linear MHD code JOREK [11]. Simulations show that a large local pellet-induced density enhancement evolves into a filament even under conditions which would otherwise not have been sufficient to generate spontaneous destabilization [12]. Hence, a sufficiently large pellet (and fuelling size pellets are always expected to be in this regime) will trigger and eventually re-introduce ELMs if a scenario is prone to ELMs at all. On the other hand, pellet injection during quiescent $\mathrm{H}$-modes $(\mathrm{QH})$ in ASDEX Upgrade did not interrupt the stationary ELM free phase [13]. This proves that conditions exists in which an H-mode with an ELM free edge barrier can be maintained even under strong pellet perturbation. The strong potential of the pellet perturbation to trigger the onset of a MHD mode is not only observed for the case of ELMs but also for neoclassical tearing modes (NTMs). For example, the threshold conditions for the onset of NTMs was mapped out by probing the stability using pellet (and saw tooth) perturbations [14]. 
Triggering ELMs by pellets is not just troublesome for the divertor heat load but also hampers the pellet fuelling efficiency. Pellet inboard launch can provide a quite suitable fuelling performance in high power discharges allowing for high density $\mathrm{H}$-mode operation. However, pellet triggered ELMs introduce enhanced particle losses during and after the deposition process. For fuelling size pellets injected into a type-I ELM phase an initial ELM is already triggered while ablation is still ongoing $[15,16]$. Moreover, in the phase following the ablation, enhanced ELM activity is observed, causing increased particle losses from the pellet particle deposition zone. If, on the other hand, large ELMs remain suppressed even in presence of pellet perturbations increased pellet fuelling efficiency could result even though additional convective energy flux remains the major pellet induced loss channel. Since pellet fuelling has already proven its ability to access plasma densities far beyond the Greenwald density $\mathrm{n}_{\mathrm{Gw}}$ [17] such a scenario could provide a high density, high confinement operation regime for ITER. The strategy for the investigation performed in the 2011 experimental campaign and reported in the following was to explore if pellet fuelling is compatible with the recently established MP ELM mitigated regimes in ASDEX Upgrade and assess if a pellet fuelled, high performance, high density regime is possible.

\section{Experimental set-up and status of coil and pellet system}

ASDEX Upgrade is a medium size divertor tokamak (major radius $\mathrm{R}_{0}=1.65 \mathrm{~m}$, minor radius $\mathrm{a}_{0}=0.5 \mathrm{~m}$, plasma current $\mathrm{I}_{\mathrm{P}}$ up to $1.4 \mathrm{MA}$, toroidal magnetic field $\mathrm{B}_{\mathrm{t}}$ up to $3.1 \mathrm{~T}$ ) with high shaping capability. All plasma facing components are completely covered with tungsten (W). The versatile set of auxiliary heating and current drive systems comprises $20 \mathrm{MW}$ neutral beam injection (NI), up to $6 \mathrm{MW}$ ion cyclotron resonance heating (ICRH) coupled power and $5 \mathrm{MW}$ of electron cyclotron resonance heating (ECRH). A fast integrated control and data acquisition system provides real time control capabilities. Proper plasma diagnosis is assured by a broad range of diagnostic systems, several of them adapted to the real time data processing and evaluation for feedback control purposes. During the 2010 vent of ASDEX Upgrade a first set of in-vessel saddle coils was installed that can produce non-axisymmetric magnetic perturbations. This is the first stage of the projected final configuration shown in the upper part of figure 1, composed of 16 B-coils (each 8 upper and lower ones, referred to as $\mathrm{Bu}-$ and $\mathrm{Bl}$-coils, respectively) and $8 \mathrm{~A}$-coils. The present coil configuration (shown in red in figure 1) consists of two rows of 4 coils evenly spaced toroidally and mounted to the passive stabilization loop on the low field side. The coils have five turns each and create a mainly radial field with toroidal mode numbers up to $n=2$ with either even or odd parity of the upper and lower coil currents. All of the experiments presented in this work were performed using this coil set.

To perform pellet fuelling studies the decommissioned high speed inboard launching system based on a centrifuge accelerator and a looping transfer system was revitalized. A sketch of the system is displayed in the lower part of figure 1. The system is currently capable of delivering pellets with a nominal particle content ranging from $1.5-3.7 \times 10^{20} \mathrm{D}$ in the velocity range $240-560 \mathrm{~m} / \mathrm{s}$ from the magnetic high field side of the torus with repetition rates of up to $47 \mathrm{~Hz}$. Within a given pellet train launched into a discharge both pellet speed and size are fixed. However repetition rates can be changed to a fixed fraction of the centrifuge revolution frequency. The pellet observation system was also refurbished and upgraded to include two ultra fast CMOS cameras and is now capable of fast individual pellet tracking. The EDICAM [18] can record a full $1024 \times 1024$ pixel image with 450 frames/s or image at a higher rate (up to $100 \mathrm{kframes} / \mathrm{s}$ ) with an accordingly reduced image size. The PHOTRON SA5 [Footnote] records $1024 \times 1024$ pixels at 7500 frames/s or up to $1 \mathrm{Mframe} / \mathrm{s}$. 

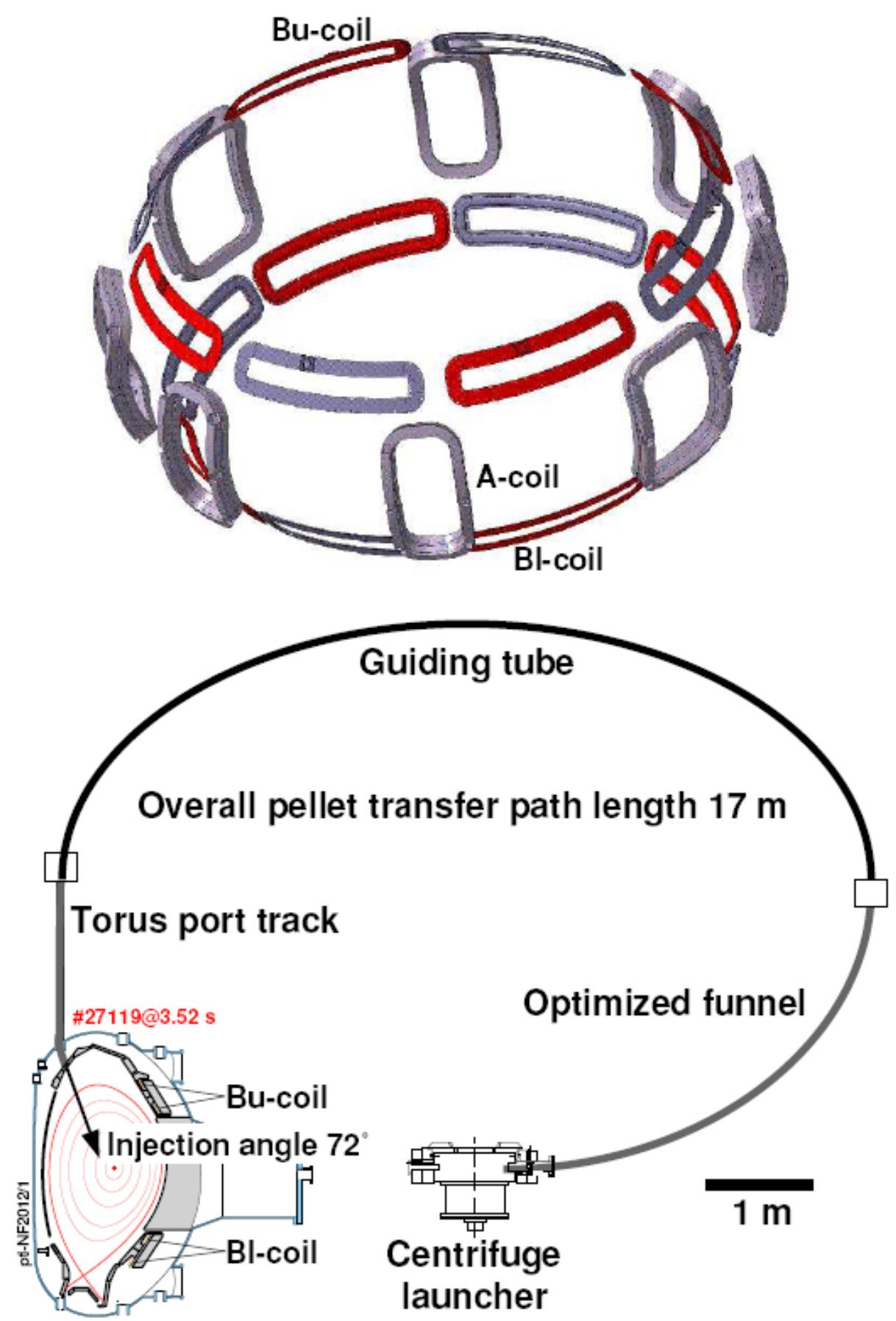

\section{ASDEX Upgrade with divertor IId}

Figure 1: Upper: Configuration of in-vessel saddle coils in ASDEX Upgrade, ultimately consisting of 8 A-coils and $2 \times 8$ B-coils (upper and lower) installed at the low field side. Currently available coils ( $2 \times 4$ coils, allowing yet for $n=2$ magnetic field perturbations) are shown in red. Lower: Centrifuge launcher and pellet guiding system capable of high-speed inboard launch and in vessel configuration of ASDEX Upgrade with divertor IId as operated in the 2011 campaign. Flux surfaces from a typical magnetic configuration are also shown in red.

The strong transient local perturbation caused by the pellet has a serious impact on several diagnostics and AUG control systems, which required dedicated attention and action to 
counteract. The plasma density feedback control system, as well as the low-density interlock of the auxiliary heating systems required for machine protection, usually rely on the lineintegrated density measurement provided by the DCN laser interferometer [19]. Strong local density gradients occurring during pellet ablation, however, cause refraction of the laser beam and make this measurement unreliable due to occurrence of fringe jumps. ASDEX Upgrade is equipped with a two-colour $\mathrm{CO}_{2} / \mathrm{HeNe}$ laser interferometer as well, which is not vulnerable to pellet perturbation due to its much shorter wavelength. This interferometer, however, has a much higher noise level compared to the DCN system. In discharges with strong pellet fuelling, interlock systems were switched to the $\mathrm{CO}_{2}$ signal during the entire discharge. For pellet density feedback control experiments a more sophisticated scheme was applied: density feedback started with the DCN signals, monitored by a real-time fringe jump detection algorithm. As soon as this algorithm detects a jump, which usually coincides with the arrival of the first large pellet at the plasma boundary, the DCN measurement is labelled invalid and the feedback controller switches to the $\mathrm{CO}_{2}$ signal. This way, the DCN measurement with its lower noise level can still be used in the plasma ramp-up phase. Although safe plasma operation was possible this way, integrated operation of diagnostics and control schemes [20] had to be applied for experiments with combined gas and pellet fuelling, e.g. feedback controlled action responding to the neutral gas pressure $n_{0}$ rather than to $n_{e}$. Moreover, information on $n_{e}$ as measured by the DCN based system is indispensable to obtain information e.g. on the exact pellet quality, the peripheral density and the density profiles. To this end, fringe jumps are corrected in post-discharge data analysis. The temporal evolution of the $\mathrm{CO}_{2}$ signal, which is much less prone to fringe jumps, was used to defer the fringe jumps of the DCN measurements. The most reliable results were achieved by applying integrated data analysis (IDA) taking into account the full set of measurements and determining the most likely corrections to all data simultaneously. IDA is based on a probabilistic description of the measured data including absolutely calibrated measurement errors, a forward model for the simulation of the data, given a density profile, and prior information about weak constraints on monotonicity. The density profile is parametrized by cubic spline polynomials [21]. Details about the application of IDA at ASDEX Upgrade can de found in [22]. Nonetheless, in some cases reliable data correction could not be achieved.

A further consequence of the larger fuelling size pellets was the safety "switch off" of the ECRH power, that often occurred. Due to the strong localized 3D density perturbation a large transient spike of reflected ECRH radiation, beyond the trigger level of the protection sniffer probe, was produced triggering a safety stop of all gyrotron action. A new scheme was developed notching the gyrotron power during the pellet impact under control of the discharge control system (DCS). Receiving information on pellet launch times and speeds the DCS calculates expected pellet arrival times at the plasma boundary. Immediately before pellet arrival (actuation starts $3 \mathrm{~ms}$ before expected pellet arrival) the ECRH power is switched off, $6 \mathrm{~ms}$ after pellet arrival the initial power is restored. All systems involved have been upgraded and operation with the improved system was successfully tested. Unfortunately, the "ECRH notching" mode became operational only at the very end of the campaign and was not yet used in the work presented.

\section{Reference scenarios}

This section provides a brief description of the ELM mitigated reference scenarios and the basic features observed in this regime. A detailed description and discussion can be found elsewhere [6]. Magnetic perturbations with $n=2$ have so far been found to result in reproducible and robust ELM mitigation in a wide heating power and safety factor range. Plasmas were run in lower single null divertor configuration with $\mathrm{I}_{\mathrm{P}}=0.8$ or $1.0 \mathrm{MA}$ and $\mathrm{B}_{\mathrm{t}}$ between $-2.3 \mathrm{~T}$ and $-2.7 \mathrm{~T}$, corresponding to edge safety factors $\mathrm{q}_{95}$ ranging from -4.8 to -6.2 . Mitigation of large type-I ELMs was achieved with the $n=2$ coil configuration with heating 
powers 2-8 times above the H-mode threshold [7,23]. For $\mathrm{I}_{\mathrm{P}}=0.8 \mathrm{MA}$ ELM mitigation is established for peripheral densities above ${ }^{-\mathrm{n}^{-} \text {e,edge }}=4.8 \times 10^{19} \mathrm{~m}^{-2}$ while $6.5 \times 10^{19} \mathrm{~m}^{-2}$ is the threshold found in the case of $\mathrm{I}_{\mathrm{P}}=1 \mathrm{MA}$, corresponding in both cases to ${ }^{-\mathrm{n}^{-}}{ }_{\text {e,edge }} \approx 0.65 \mathrm{xn}_{\mathrm{Gw}}$ [7]. So far it is not possible to distinguish whether the minimum density requirement corresponds to an edge collisionality threshold or a critical fraction of the Greenwald density limit. Once the mitigation regime is properly established, type-I ELMs disappear completely and are replaced by small ELM-like events. There is no continuous evolution of ELM energy loss or peak divertor power between large and small ELM events. At the same gas fuelling rate, the density in the mitigated phase is similar to or higher than in the preceding type-I ELMy phase; a field perturbation induced "density pump-out" is not observed. This is a marked difference to other experiments, e.g. in DIII-D [8] and JET [3] where the density reduces when the magnetic perturbation is applied and additional fuelling is required to compensate.
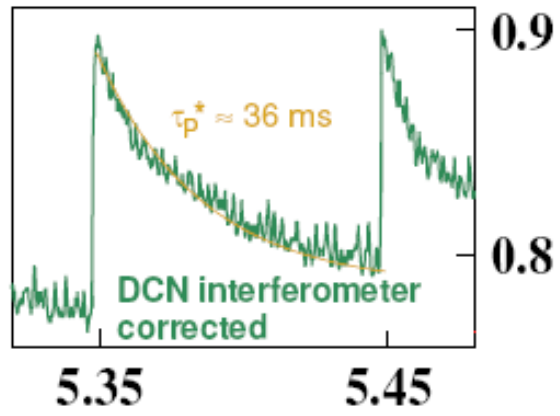

\#26079

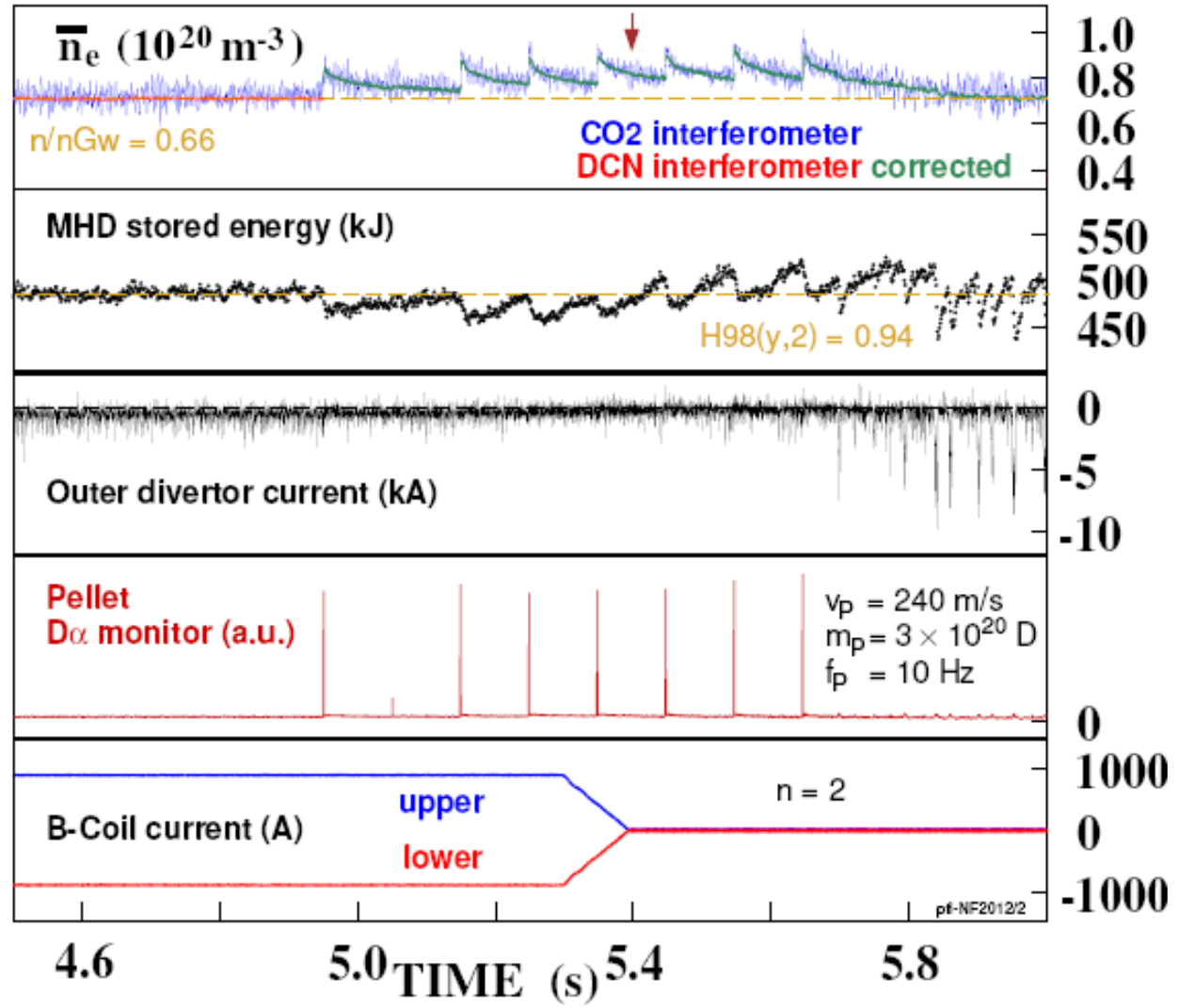

Figure 2: Slow fuelling size pellets (largest achievable perturbation magnitude) at the tailend of a MP generated ELM mitigated phase. Neither during phases with full or ramped down coil current nor in the following phase with sustained ELM mitigation do pellets trigger ELMs. Instead, they show a high fuelling efficiency and a smooth density relaxation after each pellet induced density jump (top insert on expanded time scale). 
For the pellet related investigations in the MP mitigation regime reported in the following, two different reference discharges were employed. Both relied on an "edge-optimized" configuration (EOC) with $\delta \mathrm{u}=0.13$ and plasma boundary conforming to the outer protection limiter shape. The odd $n=2$ coil parity configuration was applied, i.e. opposite polarity of upper and lower coils. All cases have $\mathrm{B}_{t}=-2.5 \mathrm{~T}$ and $\mathrm{I}_{\mathrm{P}}=0.8 \mathrm{MA}$ or $\mathrm{I}_{\mathrm{P}}=1.0 \mathrm{MA}$, giving $\mathrm{q}_{95}$ $\sim 5.6$ and 4.5, respectively. In the 1.0 MA discharge this constitutes a maximum resonance case, but using $0.8 \mathrm{MA}$ still creates a resonant perturbation.

\section{COMPATIBILITY OF MP ELM MITIGATION AND PELLET FUELLING}

The compatibility of fuelling size pellet injection with magnetic perturbation induced ELM mitigation is demonstrated in the current flat-top phase with stable ELM mitigation of the $\mathrm{I}_{\mathrm{P}}=$ 0.8 MA case shown in Fig. 2. A pellet train covers the end of the coil current steady state phase, the coil current ramp down $(100 \mathrm{~ms})$, and also the phase immediately afterwards showing sustained ELM mitigation. After termination of the coil current a slow gradual increase of the plasma stored energy sets in, lasting several hundred ms. Finally, the level of type-I activity is approached and type-I ELMs reappear. As can be seen by the absence of large ELM-induced divertor current spikes (Fig. $23^{\text {rd }}$ trace from top), neither in the ELM mitigated phase with activated coils nor in the following sustained phase do pellets trigger strong type-I ELMs. The example in figure 2 displays a pellet sequence consisting of 8 pellets (the second pellet is very small) where the 7 large pellets impose the strongest local perturbation attainable with the available launching system. The pellets exhibit remarkably good fuelling behaviour. Since no initial ELM is triggered, high fuelling efficiency (estimated $70-100 \%$ of the arriving pellet mass) is achieved by the pellet particle deposition followed by a smooth density evolution. Little impact is observed on confinement which quickly recovers after every pellet.

To emphasize the preservation of ELM mitigation and the resulting improved fuelling behaviour, a pellet launched into a MP ELM mitigated phase ( $\left({ }^{\text {rd }}\right.$ pellet in train from Fig. 2$)$ is compared to one launched into an otherwise equivalent type-I ELMing case (no coil activation). Figure 3 shows the impact of these pellets on the line average density, plasma stored energy, and diverter power load. Due to the low pellet rate in both cases $(10 \mathrm{~Hz})$ each pellet can be regarded as an individual, temporary perturbation since the plasma (almost) returns to the initial conditions before the next pellet's arrival.

The pellets compared in Fig. 3 are large and slow in order to provide the maximum possible edge perturbation. They do not represent the best fuelling approach. The pellet arriving during a type-I ELMing phase (\#26064, left hand side of figure 3) most likely triggers the ELM which occurs at the same time. The pellet launched into the MP mitigated phase causes significantly less impact on the MHD activity in the edge region. No strong ELM is triggered. In both cases, of course, the massive particle deposition in the outer plasma region has strong impact on the plasma. Pellets cause a strong increase of the peripheral density and a corresponding reduction of temperature at the pedestal top, as well as a short transient drop in toroidal rotation. Both pellets show very similar ablation behaviour; the estimated penetration depth along the designated path inside the separatrix is about $0.3 \mathrm{~m}$. In the non-mitigated case since type-I ELMs occur while pellet ablation is still ongoing the density increase after complete pellet ablation is somewhat reduced with respect to the MP-mitigated counterpart. An even more pronounced difference is observed in the post pellet density evolution. While pellet deposited particles are very rapidly lost due to the type-I ELM activity eroding the deposition profile, in particular close to the edge, a smooth decay and a more persistent density elevation is observed for the MP case. In both cases the post pellet phase is characterized by an enhanced particle flux from the plasma edge. Since particles are deposited with virtually no thermal energy but are leaving the plasma after having undergone thermalization this results in additional convective energy losses. The losses cause a weak 
transient reduction of the plasma energy and accordingly a slight enhancement of the steady state power flux into the divertor. In the type-I ELMing case these effects are overlaid by the much stronger variations during the ELM cycle whereas in the MP mitigated scenario the pellet effect is much clearer, especially with respect to the stored energy and the peak power flux lost to the divertor. Pellets in the MP mitigated phase do not result in significant energy losses and power fluxes even for the scenario presented here, which features pronounced particle deposition at the edge. Therefore, applying more fuelling-like pellet parameters (in particular, higher pellet velocity) will result in deeper penetration and particle deposition and can be expected to further improve the fuelling performance.

It should be noted finally that pellets in the MP mitigated scenario have a more pronounced impact on the core MHD activity. Increased plasma inertia slows down the main $n=1$ mode present in the plasma and transiently gives rise to an additional $n=1$ "satellite" mode at lower frequency although pellets certainly do not penetrate as far as the $\mathrm{q}=1$ surface. Dedicated investigation on this effect are ongoing, however the matter is beyond the scope of this report and will be presented elsewhere.

Hence, it is concluded that in the operational regime found for MP-induced ELM mitigation, which is characterized by sufficiently high peripheral density, pellet fuelling is a suitable fuelling approach since reappearance of large type-I ELMs does not take place. Even for the strongest pellet perturbations possible with the available launching system and under conditions in which spontaneous type-I ELM activity would otherwise be present, no strong ELM is triggered. In addition, excellent fuelling behaviour was found as a direct result of the complete absence of large ELMs. Consequently, further experiments were performed to study the effectiveness of pellet fuelling in MP ELM mitigated scenarios (see section 6).

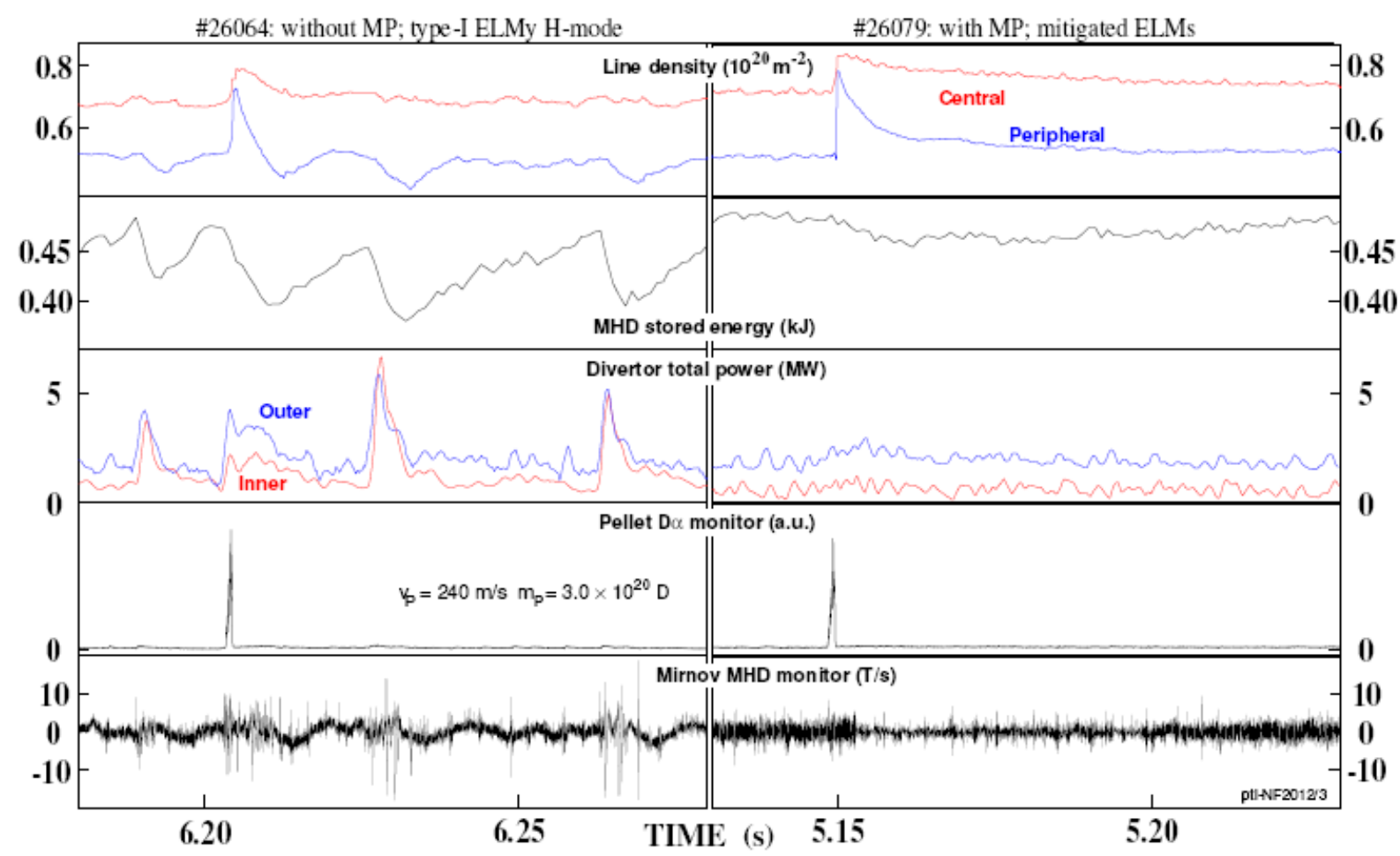

Figure 3: Comparison of virtually identical cases of pellet injection (pellet and plasma parameters) into H-mode discharges without (left part) and with (right part) MP. Mitigated small high frequency ELMs occur with MP coils on while the reference case shows distinct strong type-I ELMs. The pellet arriving in the mitigated phase results in better and longer lasting density enhancement while energy losses and power fluxes to the inner and outer divertor do not reach the same magnitudes as in the type-I ELM case. 


\section{Pellet assisted access to the MP ELM mitigated regime}

As an initial fuelling application, pellets were employed to assist access to the ELM mitigated regime in a case with $I_{P}=0.8 \mathrm{MA}$ while operating with freshly boronized walls. Due to the high wall pumping under such conditions the requested peripheral densities are hard to establish. The massive gas bleeding required would result in unwanted gas loading of the wall. As can be seen in figure 4, pellets are able to establishing easily the required conditions. All three discharges shown here have identical plasma parameters with coil activation and strong gas puffing to achieve the required operational condition. Whereas under standard operational conditions (unboronized walls, \#26081, lower panel Fig. 4) the gas puff is sufficient to reach the peripheral line density, a freshly boronized vessel causes strong wall pumping and full suppression of type-I ELMs can not be established (\#26114 middle panel). However, for appropriate initial plasma parameters the sudden pellet initiated density step is able to kick the edge into the ELM mitigation regime even with boronized walls (\#26115 upper panel). To optimize edge fuelling, large pellets at a speed of $240 \mathrm{~m} / \mathrm{s}$ were employed at a modest $10 \mathrm{~Hz}$ repetition rate. For this repetition rate, during the early part of the pellet train the induced density enhancement drops back below the critical density level (indicated by horizontal lines in figure 4) and type-I ELMs re-occur until the arrival of the next pellet. Persistent mitigation is achieved once the frequency of the pellets is sufficiently high to keep the edge density above the threshold level. During and after the pellet phase in \#26115 density measurements from DCN interferometer required fringe jump corrections introducing some uncertainty.

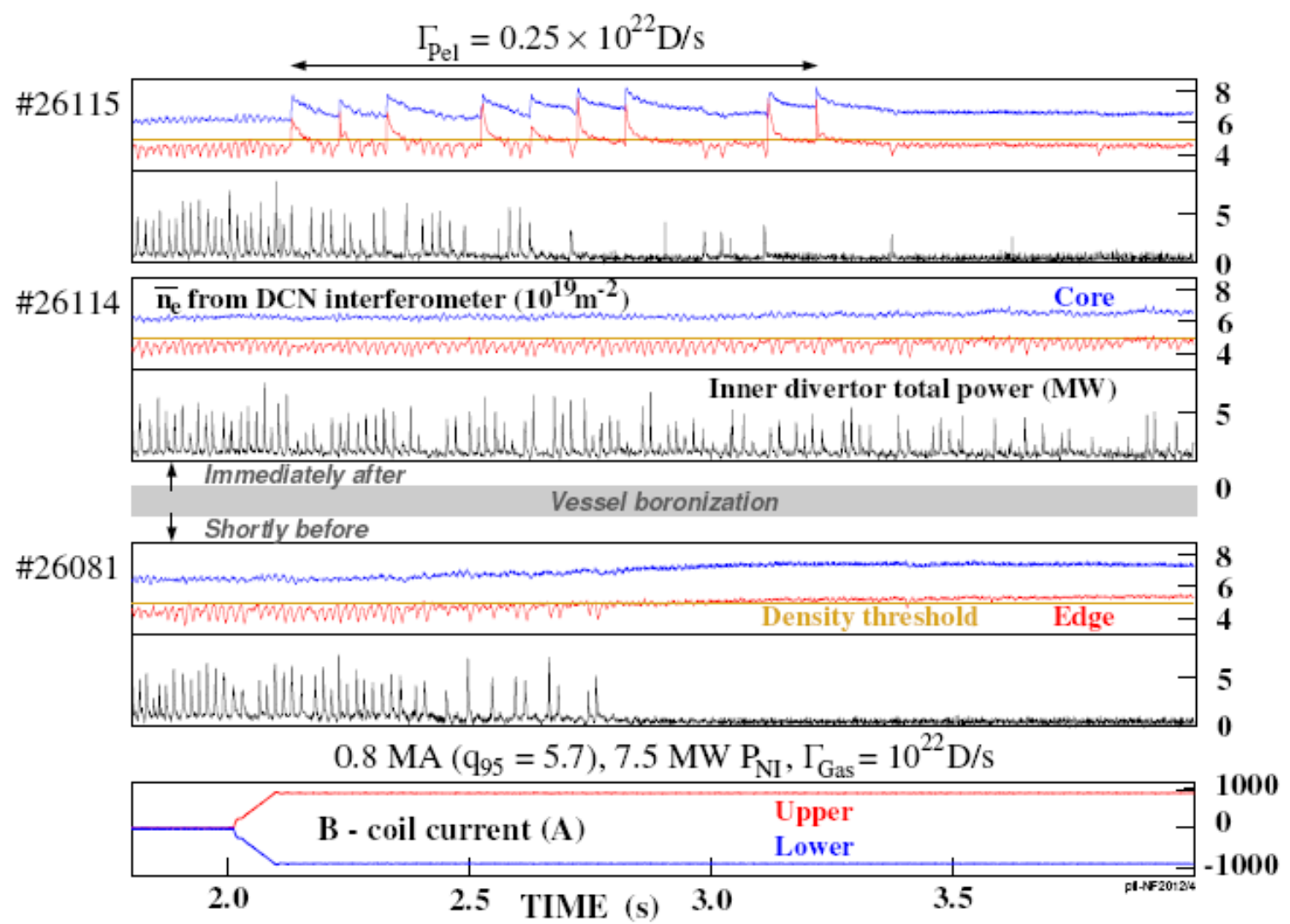

Figure 4: Pellet fuelling assisted access to the MP ELM mitigated regime. All discharges have identical plasma parameters and gas puffing. \#26081: gas puff sufficient to reach threshold density. \#26114: in freshly boronized vessel gas puff becomes insufficient. \#26115: adding pellets kicks the discharge into the mitigation regime. The empirical peripheral density mitigation threshold is indicated. 


\section{Pellet fuelled high density operation in the MP ELM mitigated regime}

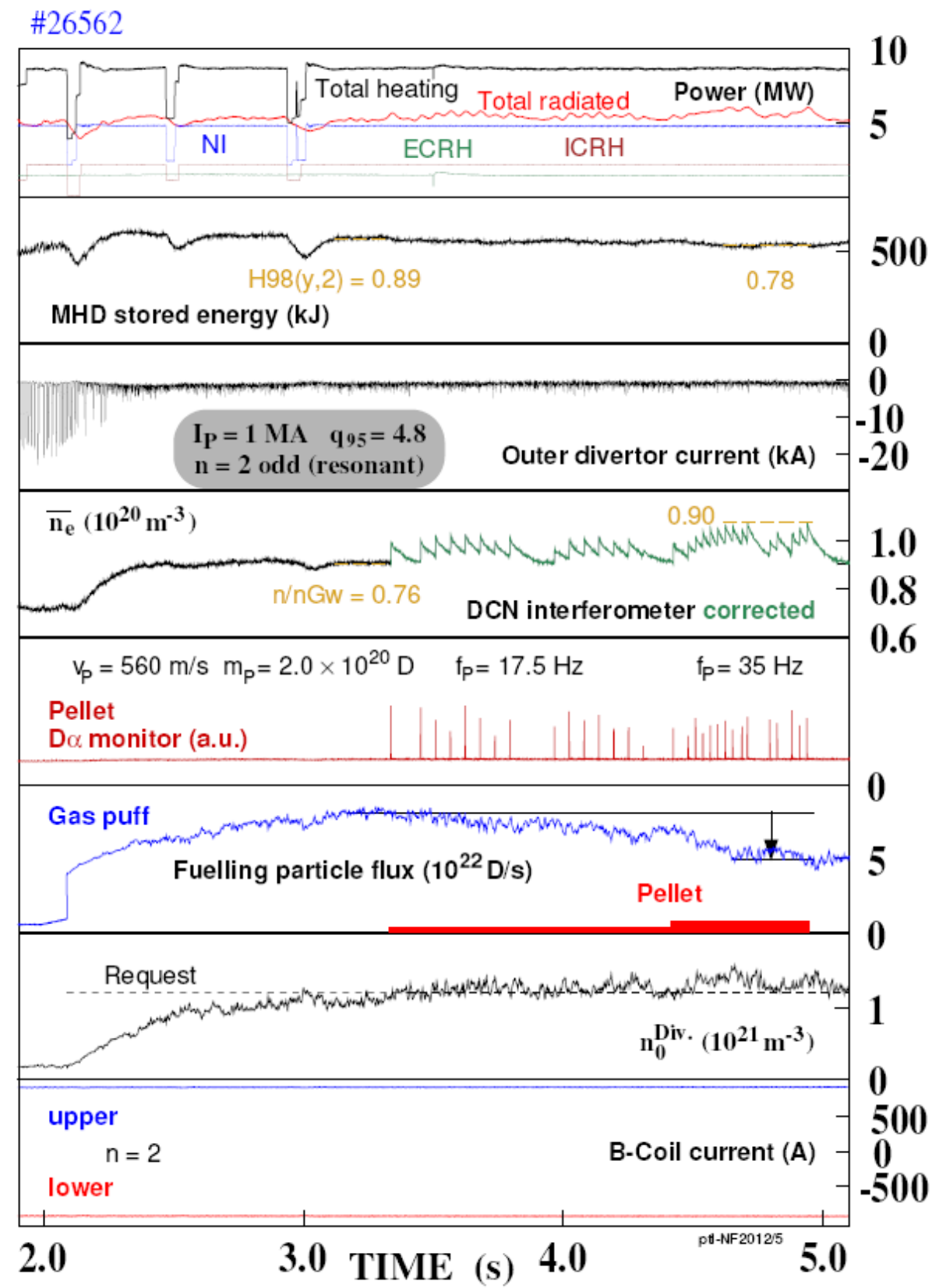

Figure 5: Density ramp up by fuelling pellets with increasing frequency. To ensure stable MP ELM mitigation a minimum divertor neutral gas pressure is kept - the required gas puff is reduced by more than 5 times the applied pellet particle flux.

In order to achieve an overall optimized performance, the gas puffing required to reach ELM mitigation can be largely replaced by the more efficient pellet fuelling. However, experiments aimed at determining the minimum amount of gas puffing required to maintain MP ELM mitigation showed that the gas puff can not be entirely replaced by pellets; with too strong a reduction causes type I ELM activity to reappear. Using the pellets a dedicated fuelling experiment was performed to achieve higher densities in order to expand the operational space of the MP ELM-mitigated regime and to explore if there is an upper limit in density.

With increasing plasma current and heating power, significantly higher gas puffing has to be applied to reach the required peripheral densities. However, strong gas puffing can cause 
confinement degradation and possibly a transition into the type-III ELM regime. This makes plasma operation more delicate at higher $\mathrm{I}_{\mathrm{p}}$ and heating power. Pellets can be applied to ease access to the ELM-mitigated regime by achieving the required edge densities while minimizing the requested gas puff. This has been done for the high current, $\mathrm{I}_{\mathrm{p}}=1.0 \mathrm{MA}, \mathrm{MP}$ ELM mitigation scenario with strong auxiliary heating (mix of neutral beam and central electron and ion cyclotron resonance heating). For this scenario mid-sized (nominal mass 2.9 $\mathrm{x} 10^{20} \mathrm{D}$ ) pellets at $560 \mathrm{~m} / \mathrm{s}$ were used for the approach to the critical density as shown in figure 5. The pellet particle flux was adjusted by the pellet frequency and doubled for the second part of the pellet train. Valves are normally feedback-controlled on the peripheral DCN interferometer line density measurement. Since pellet induced fringe jumps distort the density measurement, this approach had to be modified. The neutral gas density in the private flux region, $\mathrm{n}_{0}{ }^{\text {Div }}$, as measured by an internal pressure gauge was found to be insensitive to pellet perturbations and in reference discharges well correlated with the peripheral density. Hence, it was taken instead as the control parameter with the requested value of $1.2 \times 10^{21} \mathrm{~m}^{-3}$ found to be appropriate in purely gas fuelled discharges to guarantee operation well above the critical edge density.

With the pellet flux pre-programmed, successful fuelling replacement can be seen as a reduced gas flux request. The estimated averaged real pellet flux arriving in the plasma, taking into account transfer losses, is shown in figure 5 . The total gas puff reduction is about 5 times the applied pellet flux. Despite this reduced total fuelling particle flux the density is increased in the pellet phase with higher pellet flux, but less total flux. Hence, pellet injection shows advantageous fuelling behaviour as compared to gas puffing and allows for high density operation with less fuel consumption and less detrimental impact on the edge. In the case shown in figure 5, a maximum density of about $1.07 \times 10^{20} \mathrm{~m}^{-3}$ is achieved, corresponding to a Greenwald factor of about 0.9 . Only a very mild $(\sim 5 \%)$ reduction of the plasma energy, attributed to pellet driven convective losses, is observed. No indication of a confinement increase with density as predicted by the ITERH98P(y,2) scaling $\left(\sim \mathrm{n}_{\mathrm{e}}^{0.41}\right)$ is found [24]. Accordingly, the experimental fraction H98 $(y, 2)$ drops with respect to the value predicted by the scaling.

In the 2011 campaign experiments were performed to determine the largest possible density enhancement with the least deleterious impact on confinement. As known from earlier pellet fuelling experiments, confinement is best preserved at high density when pellet induced convective losses are minimised. The preferred approach to density enhancement with a pellet particle flux as low as possible consequently aims at maximising pellet particle sustainment time in the plasma [16]. This can be achieved by the deepest possible pellet penetration and deposition, i.e. maximised pellet mass and speed for any given set of plasma parameters. However, for an inboard launch system that relies on transfer systems with a technically fixed maximum launch mass the transfer loss increases with pellet velocity. This leads to a roll over of fuelling performance with increasing $\mathrm{v}_{\mathrm{P}}$ since the $\mathrm{m}_{\mathrm{P}}$ arriving at the separatrix shrinks and so does the penetration depth. Hence, although the AUG pellet system in principle is capable of operating at speeds up to $1000 \mathrm{~m} / \mathrm{s}$, the best and maximum fuelling conditions are achieved using the largest available pellet size at $\mathrm{v}_{\mathrm{P}}=560 \mathrm{~m} / \mathrm{s}$. The maximum repetition rate for reliable and sustained operation is $47 \mathrm{~Hz}$. The same plasma scenario as was used for the gas puff replacement experiment (see figure 5) was chosen to find the maximum pellet driven density enhancement possible while maintaining good plasma confinement. For this experiment a pre-programmed gas puff just sufficient to establish ELM mitigation was used. As shown in figure 6 after a first initial reference phase a sequence of maximum pellet fuelling lasting for about $0.85 \mathrm{~s}$ was added. With the pellet train established, the gas puff rate was reduced from $5.2 \times 10^{20} \mathrm{D} / \mathrm{s}$ to $3.7 \times 10^{20} \mathrm{D} / \mathrm{s}$ to keep the total particle flux constant. The perturbation by the second pellet caused cut-off and subsequent tripping of the ECRH. The reduced heating power results in a drop of the stored energy. The ongoing pellet flux results in 
a gradual build up of the line averaged density with only a marginal influence on the stored energy. At the end of the maximum pellet flux phase a value of $\mathrm{n}^{-} \mathrm{e}=1.8 \times 10^{20} \mathrm{~m}^{-3}$ is achieved, corresponding to a Greenwald factor of 1.5. During this entire high density phase the ELMs remain mitigated. So far this is the highest density obtained with ELM mitigation, and it is limited only by the present technical limitations of the pellet fuelling system. Once again, the energy confinement does not follow the ITERH98P(y,2) scaling prediction. At the end of the high frequency pellet train a slow ramp-down of the coil current is initiated with the gas puff rate kept at the reduced level. Two more pellets at $9 \mathrm{~Hz}$ rate are injected in order to probe the edge stability. Type-I ELMs return already in the phase between these two pellets and the second probing pellet triggers the first strong ELM. Finally, an unstable type-I ELMy $\mathrm{H}$-mode phase is established with the density returning to its initial value and the applied heating power identical to the high density phase. As indicated in figure 6, a second reference period is taken from this phase. The example shown in Fig. 6 was found to be reproducible and demonstrates high density operation far beyond the Greenwald density without deleterious impact on confinement when using pellet fuelling.

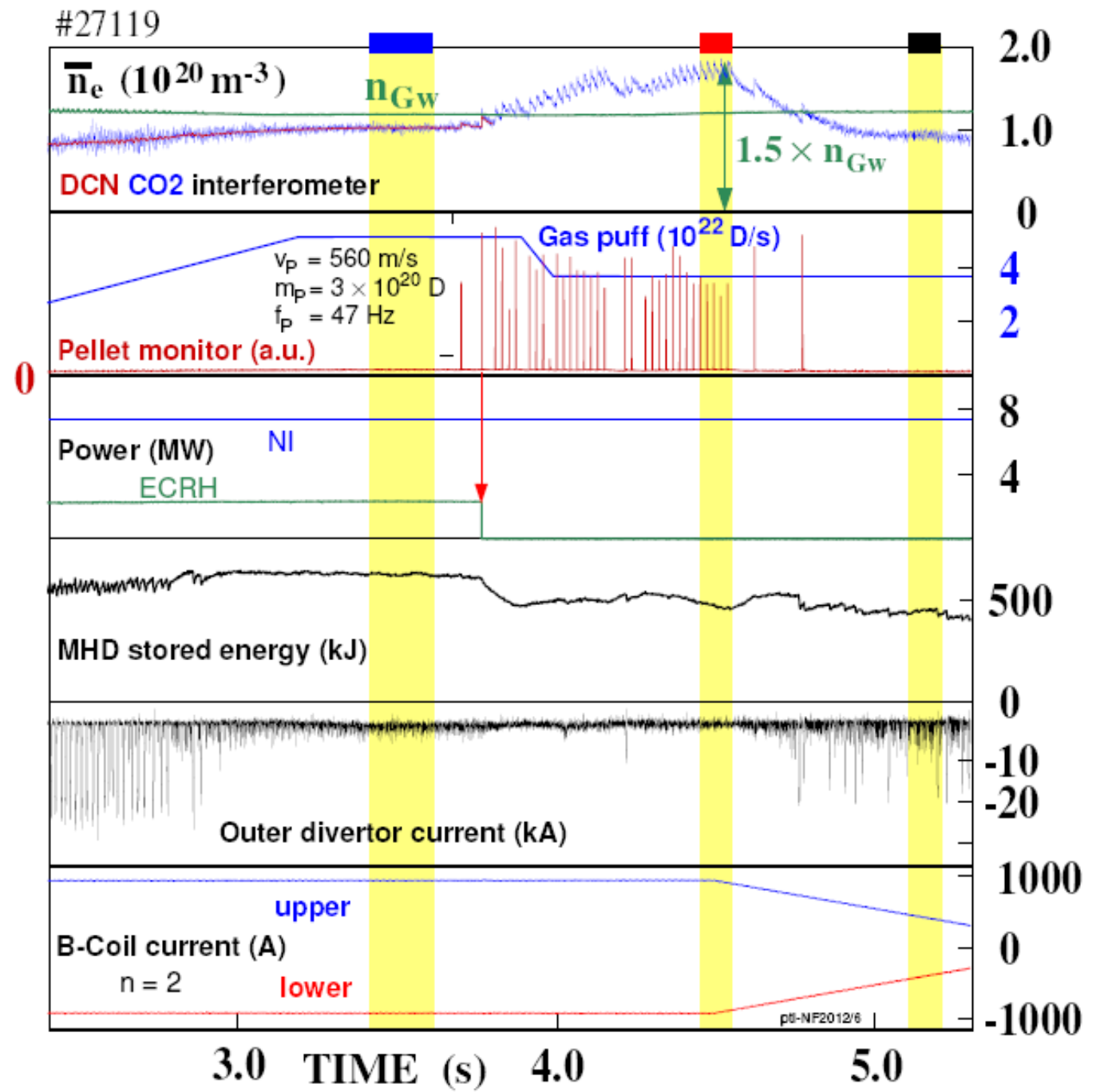

Figure 6: Demonstration of MP ELM mitigated operation at densities far beyond the Greenwald density by pellet fuelling. After reaching the mitigation regime by B-coil activation and sufficient gas puffing, maximum available pellet fuelling is applied. The gas puff reduced accordingly. Pellet fuelling gradually increases the line averaged density to about 1.5 times the Greenwald density without significant impact on confinement (the drop in energy is due to the loss of ECRH) while maintaining ELM mitigation. Phases for which profiles are taken are indicated by vertical highlighted bars. 
Progressing beyond the Greenwald limit by means of pellet fuelling in AUG is achieved by overcoming the flat density profiles typically observed in high density operation with gas puffing. Pellet fuelling experiments in ELMy H-mode plasmas (with less efficient fuelling) showed that a gradual pressure/energy loss can be avoided when approaching the edge density limit and thereby, high density operation can be combined with reasonable confinement [16]. As can be seen in the density profiles shown on the right hand side of figure 7 , the density increase during the pellet train takes place entirely inside the pedestal top while the pedestal itself remains, within diagnostic resolution, unchanged. The profiles shown are obtained by applying Integrated Data Analysis (IDA) to all available validated data [21,22]. In the plasma centre the initial density is almost doubled reaching values of $\mathrm{n}_{\mathrm{e}}=2.0 \times 10^{20} \mathrm{~m}^{-3}$ (corresponding to a Greenwald factor of 1.6).
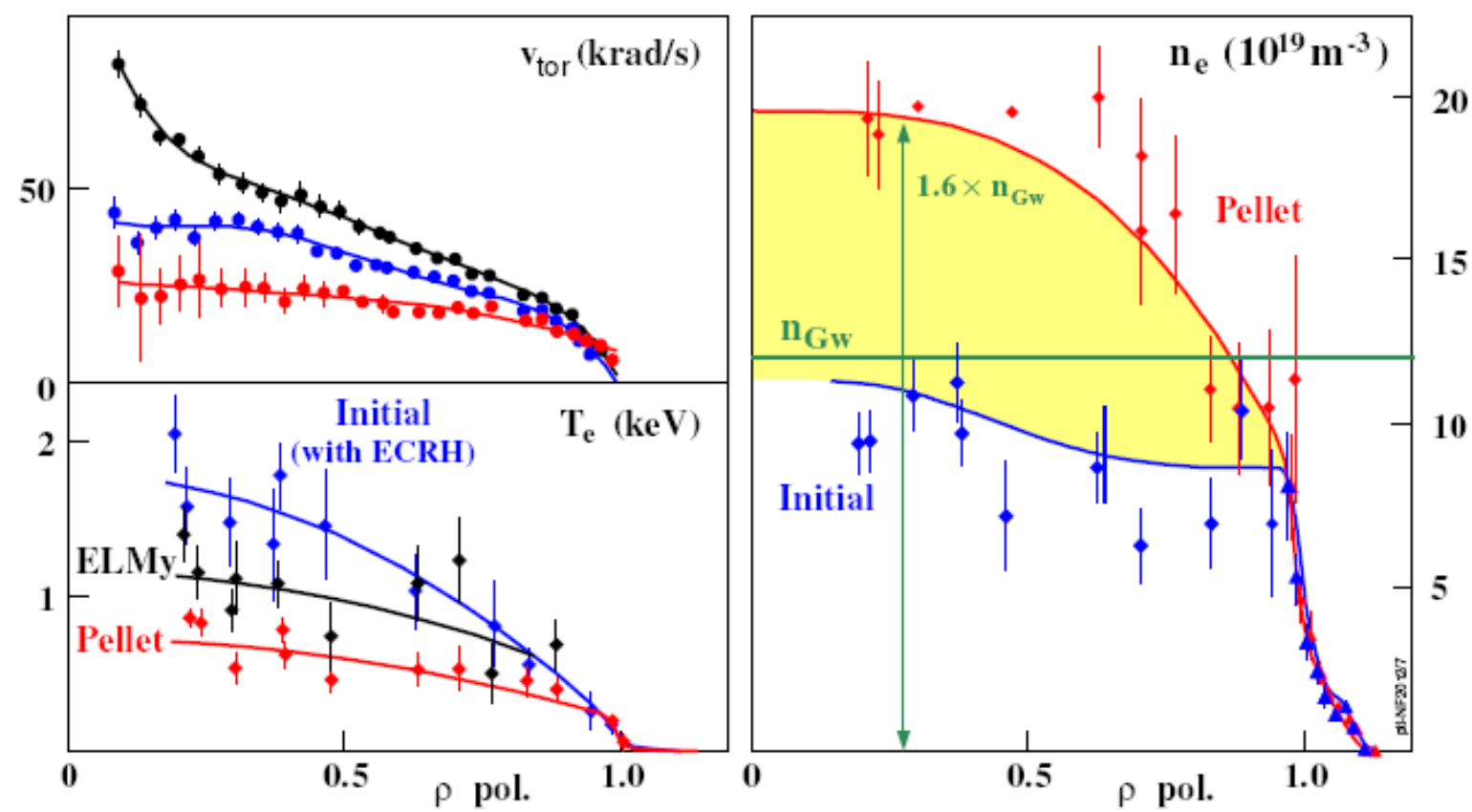

Figure 7: Profiles taken for the three phases indicated in figure 6 (initial, pellet, final ELMy). Right: density profiles (diamonds from Thomson scattering, triangles from lithium beam, both with error bars; solid lines obtained from IDA taking into account also fringe corrected DCN interferometer data) for initial and pellet phase. Left lower: electron temperature from Thomson scattering (solid lines obtained from modified tanh fit). Left upper: toroidal plasma rotation derived from CXRS using beam neutrals and B/C impurities (solid lines from polynomials fit).

The temperature and plasma rotation profiles, like the density, show no significant change at the pedestal top as can be seen on the left hand side of figure 7. The electron temperature $\left(T_{e}\right)$ pedestal profiles as measured by the Thomson scattering diagnostic agree within the data resolution for all three phases. An enduring effect of the strong pellet fuelling is only observed inside the edge transport barrier. The pellet and final ELMy phase, which are subject to the same heating power, show almost identical energy content and electron pressure profiles. Accordingly, the $\mathrm{T}_{\mathrm{e}}$ profile in the high density pellet phase is significantly reduced with respect to the ELMy phase. Due to the central ECR heating applied in the beginning of the discharge, the core $T_{e}$ is higher in the initial phase than in either the pellet or ELMy phases. Ion temperatures $\left(\mathrm{T}_{\mathrm{i}}\right)$ were measured employing several independent charge exchange recombination spectroscopy (CXRS) systems using neutral heating beams. During the pellet phase $T_{i}$ is found to be approximately equal to $T_{e}$ due to high collisionality. For both the initial and the ELMy reference phases very similar $T_{i}$ profiles were measured reaching about 
$1.5 \mathrm{keV}$ in the centre. The core toroidal rotation is significantly reduced during the pellet phase due to the increase in the plasma density (mass) and the corresponding changes in the NBI torque deposition profile. This is shown in the upper left part of figure 7. This effect is most pronounced when comparing the pellet and ELMy phase, which employed the same heating configuration. Here, the plasma rotation is reduced by a factor of two while the plasma density is increased by the same factor. The rotation in the initial mitigated phase is reduced with respect to the ELMy phase due to the ECRH which increases the core momentum diffusivity [25].

\section{Confinement at high densities and limitation of the ITERH98P(y,2) scaling}

The quality of the confinement achieved in a plasma scenario is assessed by taking the ratio of the energy confinement time to the value predicted by the ITERH98P(y,2) scaling. It is a well known fact from ITER confinement database studies [26] that for densities near and beyond the Greenwald value the confinement observed no longer agrees with the scaling and the corresponding H98 values drop significantly below unity. Pellet fuelling experiments performed at JET and AUG showed that in ITER relevant scenarios a density regime beyond values accessible with gas puffing can be accessed but there is no further improvement in the energy confinement [27]. In these experiments the observed energy confinement behavior could be modeled well by taking into account the initial energy confinement and the subsequent losses from thermalized pellet fluxes. Therefore, if the pellet induced convective losses can be avoided it should be possible to at least maintain the initial confinement in a high density pellet fuelled scenario. As mentioned in section 6, similar behavior was again found in this study. Access to the high density regime is possible but no confinement increase as predicted by the ITERH98P $(\mathrm{y}, 2)$ scaling is found.

From reference plasmas and pellet fuelling experiments using the 1.0 MA scenario with different heating power levels, the ratio $\mathrm{H} 98(\mathrm{y}, 2)$ of achieved $\tau_{\mathrm{E}}$ to the value predicted by the ITERH98P $(\mathrm{y}, 2)$ scaling was calculated using the thermal energy confinement time, i.e. excluding fast ion contributions and taking into account heating power losses [28]. It shows that type-I ELMy reference phases are characterized by $\mathrm{H} 98(\mathrm{y}, 2)$ values between 0.8 and 0.85 , increasing slightly when entering the mitigated phase at about ${ }^{-} \mathrm{n}^{-}{ }_{\mathrm{e}}=0.7 \mathrm{x} \mathrm{n}_{\mathrm{Gw}}$ (with $\mathrm{n}_{\mathrm{Gw}}$ $=1.2 \times 10^{20} \mathrm{~m}^{-3}$ ). Reference phases with pure gas puff fuelling show that there is only quite a narrow density regime accessible and that in the mitigated phase the H98 (y,2) value decreases with increasing density. When pellet fuelling is applied, the plasma energy is reduced by about $5-10 \%$ when progressing beyond about $0.75 \times \mathrm{n}_{\mathrm{Gw}}$ to about $0.9-0.95 \times \mathrm{n}_{\mathrm{Gw}}$. Once this initial energy reduction has taken place, no further significant confinement $\left(\tau_{\mathrm{E}}\right)$ degradation is found with advancing to even higher densities as can be seen from figure 6 .

\section{ELM trigger potential comparison: ELM-mitigated and type-I regimes}

The potential of non-axisymmetric magnetic perturbations for the suppression of ELMs becomes obvious when considering the pellet-induced perturbation as a probe for edge stability. While even the strongest pellet imposed perturbations do not trigger ELMs under appropriate MP suppression conditions as shown and discussed in section 4, much weaker perturbations can trigger type-I ELMs in discharges without coil activation even very early in H-mode phases, before the edge pedestal would naturally be susceptible to type-I ELM instabilities. The latter is shown in figure 8 , which displays a train of pellets launched into the plasma in the absence of MP during an L-H transition. Pellets reaching the plasma immediately after the transition trigger ELMs despite the edge pedestal not being fully formed and still far from saturation of edge pedestal pressure. Triggering an ELM right after the Hmode transition might be useful to avoid a long ELM-free phase that is often terminated by a particularly large type-I ELM. 


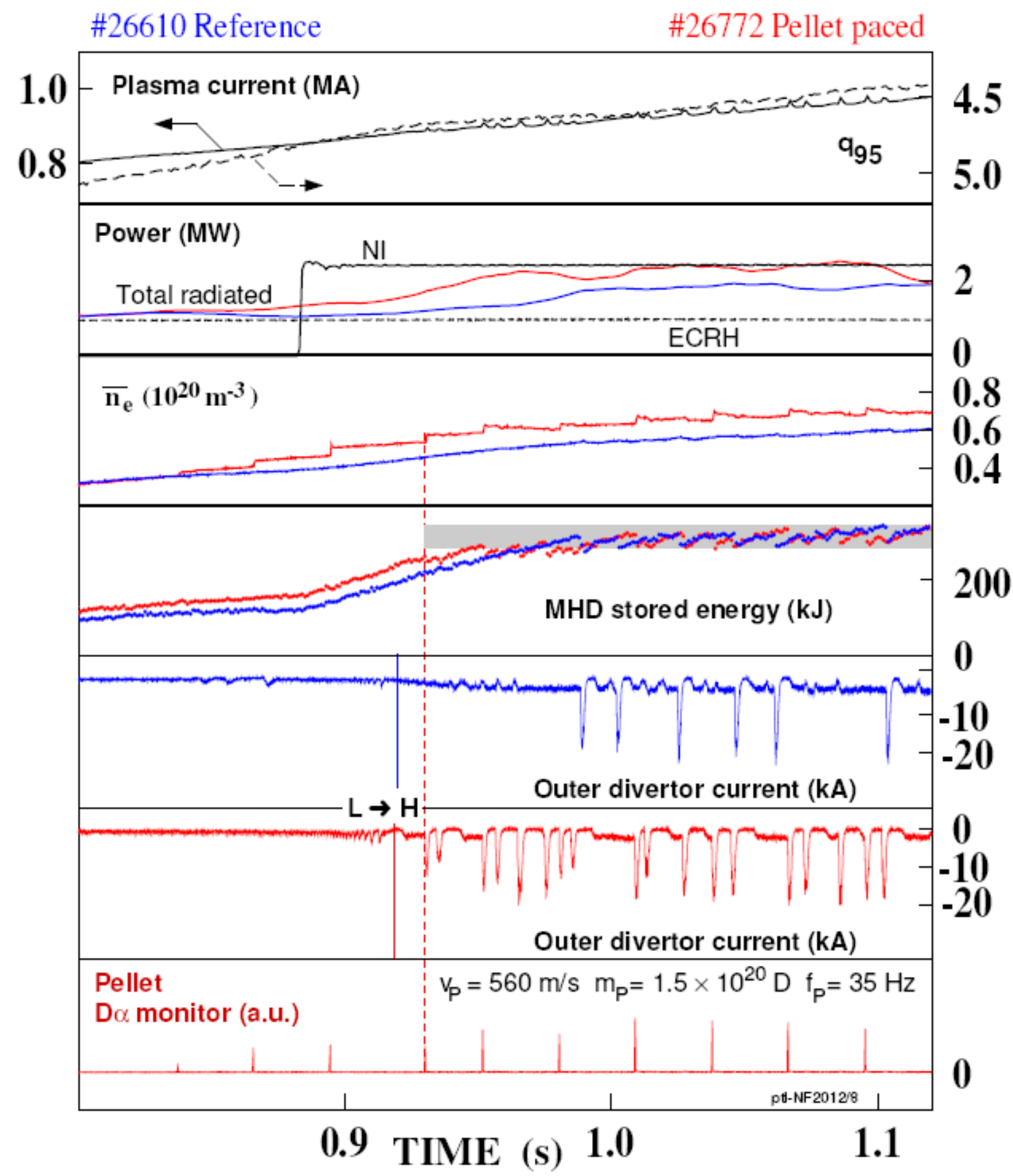

Figure 8: Pellet pacing control of the ELM activity from the onset of the H-mode. In the absence of MP coil activation pellets trigger ELMs immediately once H-mode is reached.

Cases where pellets trigger ELMs and configurations with MP induced suppression, in which they do not trigger ELMs, can be compared in detailed in order to identify crucial features and conditions for pellet ELM triggering or successful ELM suppression. A first approach of this kind was made taking advantage of the new pellet observation system's high temporal and spatial resolution. The 3D non-linear MHD code JOREK [29] predicts the evolution of the pellet ablation plasmoid into a first ELM filament in at least qualitative agreement with the experimental observations from JET [30] and DIII-D [31]. Hence, we look for differences between the two cases in the pellet ablation phase when the plasmoid is formed around the pellet. Up to now, no differences could be recognised. Long exposure images recording the 
whole pellet trajectory showed the same penetration depth. This was expected since the target plasma electron temperature and density profiles are virtually identical in both phases. A comparison of two virtually identical pellets (Large size at $240 \mathrm{~m} / \mathrm{s}$, compare figure 4) arriving during a type-I ELMy phase and a ELM mitigated phase with almost identical plasma parameters is shown in figure 9. Whereas the earlier pellet (left picture) triggers an ELM, the later one (right picture) does not. The structure of the observed radiating cloud was also similar, no extra filamentary structure appeared at the time an ELM was triggered. Short exposure images revealed the same cloud extension both in toroidal and radial direction and also the detached cloudlet drifting to the LFS was observed for both phases. Observations so far show pellet ablation and hence pellet induced perturbations must be very similar during according ELMy and mitigated phases. Obviously MP activation prohibits the pellet seed perturbation from evolving into a type-I ELM. More detailed investigations on the pellet ablation within the pedestal region where type-I ELMs are triggered applying the maximum temporal and spatial resolution of the systems are envisaged for the next experimental campaign.
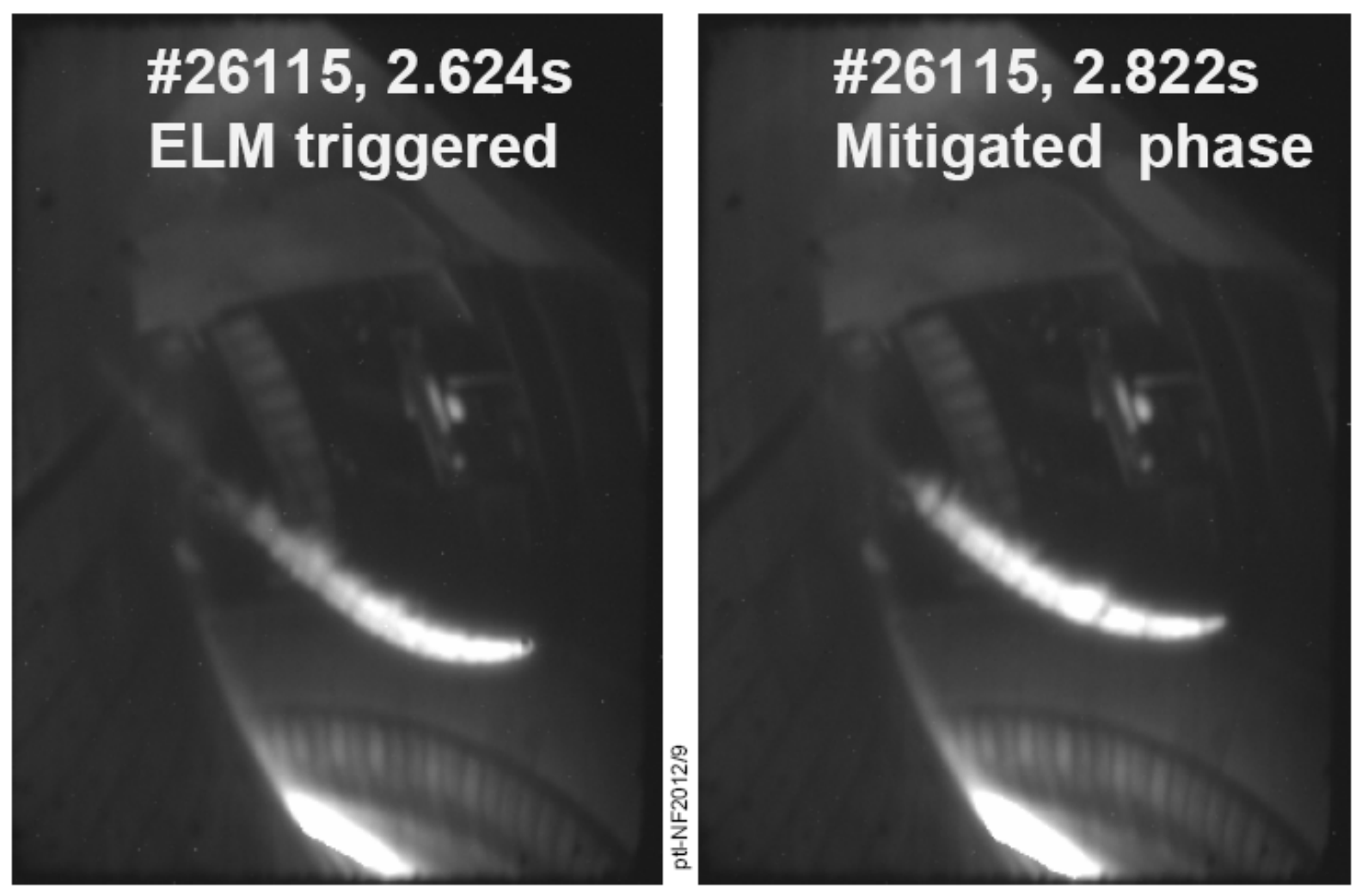

Figure 9: Long exposure images of ablation pellets during type-I ELMy and ELM mitigated phase. Pellet and plasma parameters are virtually identical. The pellet shown on the left side triggers an ELM whereas the pellet shown on right side does not.

\section{Conclusion}

Our observations demonstrate that pellet fuelling is compatible with the ELM mitigation technique that relies on non-axisymmetric magnetic perturbations. Moreover, it indicates such a scenario is even ideal for pellet particle fuelling: inboard launch takes advantage of the drift forces that accelerate the pellet ablated material towards the low field side and prompt losses due to pellet-triggered large ELMs are avoided by successful ELM mitigation. Pellet fuelling can be employed to ensure reliable access into the ELM mitigation regime, characterized at ASDEX Upgrade essentially by a threshold in the peripheral density. Pellets can extend the ELM mitigated domain towards high densities even far beyond the Greenwald density. For 
scenario optimization the gas puff rate can be efficiently replaced resulting in high density operation with a modest impact on the confinement.

A strong synergy effect can be achieved when combining ELM mitigation by MP and pellet fuelling. Pellet fuelling can assist the access to the ELM mitigation regime, extend the operational range and improve on plasma performance. In turn the absence of strong ELMs helps to avoid strong particle losses during and after the pellet ablation resulting in a more favourable fuelling behaviour. Pellets launched into ELM mitigated plasmas from the inboard side show fuelling features even under high power conditions usually observed only in a low power regime. At least advanced fuelling performance at a high heating power level helps to minimise pellet generated convective losses allowing for an even better performance in the high density regime than observed for type-I ELMy scenarios. It is worth noting that a similar synergetic effect can not be expected when employing pellets for both ELM mitigation by frequency control and plasma fuelling. In this case fuelling pellets would still be hampered by strong ELM losses while pacing pellets tailored for shallow penetration and deposition result in significant convective losses and cause a heavy pumping burden.

The ITERH98P $(\mathrm{y}, 2)$ scaling generally used for performance assessment and employed for ITER predictions includes a monotonic positive dependence of confinement on density. However, no indication was found for confinement improvement with density beyond about 0.75 - 0.8 times the Greenwald density. At the best, high densities can be achieved while maintaining the initial confinement time.

There is a remarkable potential of the magnetic perturbation to suppress the pellet ELM trigger capability. Even the strongest achievable pellet perturbations during MP phases did not trigger ELMs while much weaker perturbations trigger type-I ELMs under plasma conditions with smaller pedestal pressure without coil activation. This observation might become very useful in order to understand the impact of the coil perturbation on the plasma edge.

Investigations reported here were intended as a first exploration step for the combined operation of active ELM control by in-vessel coils and pellet fuelling, performed using just the first stage of the coil assembly and employing a pellet system still limited in technical performance. More detailed investigations using further developed facilities are planned for the upcoming campaigns. For the next campaign the full set of $\mathrm{B}$ coils will be available promising improved ELM mitigation possibilities. Also, the pellet system is being upgraded to achieve better performance with respect to fuelling features. In particular, the number of particles carried within a pellet train will be increased by extending train durations and operating at higher repetition rates. Higher pellet speeds and more flexibility of pellet parameters within a single train (different sizes, more flexible repetition rates) are also envisaged.

\section{Footnote}

PHOTRON LIMITED; http://www.photron.com/datasheet/FASTCAM_SA5.pdf

\section{References}

[1] T. E. Evans et al., Phys. Rev. Lett. 92, 235003 (2004).

[2] Y. Liang et al., Plasma Phys. Control Fusion 49 (2007) B581.

[3] Y. Liang et al., Plasma Fusion Research 5 (2010) S2018.

[4] J.M. Canik et al., Nucl. Fusion 50 (2010) 034012.

[5] A. Kirk et al., J. Nucl. Mater. 415 (2011) S910.

[6] W. Suttrop et al., Fusion Eng. Design 84, 290 (2009).

[7] W. Suttrop et al., Phys. Rev. Lett. 106, 225004 (2011).

[8] T. E. Evans et al., Nucl. Fusion 48, 024002 (2008).

[9] Y. Liang et al., J. Nucl. Mater. 390-391, 733 (2009). 
[10] P.T. Lang et al., Nuclear Fusion 48, 095007 (2008).

[11] G.T.A. Huysmans et al., Plasma Phys. Control. Fusion 51, 124012 (2009).

[12] G.T.A. Huysmans et al., in Fusion Energy 2010 (Proc. 23rd Int. Conf. Daejeon, 2010)

(Vienna:IAEA) CD-ROM file THS/7-1 and

http://www-naweb.iaea.org/napc/physics/FEC/FEC2010/html/index.htm

[13] W. Suttrop et al., Nucl. Fusion 45, 721 (2005).

[14] H. Zohm et al., Nucl. Fusion 41, 197 (2001).

[15] L.R. Baylor et al., Nucl. Fusion 47, 1598 (2007).

[16] P.T. Lang et al., Nucl. Fusion 40, 245 (2000).

[17] M. Greenwald et al., Nucl. Fusion 28, 2199 (1988).

[18] A. Szappanos, G. Kocsis, A. Molnar, J. Sarkozi, S. Zoletnik, Fusion Eng. Design 83, 370 (2008).

[19] A. Mlynek et al., Rev. Sci. Instrum. 81, 033507 (2010).

[20] W. Treutterer et al., Fusion Eng. Design 86, 465 (2011).

[21] R. Fischer, E. Wolfrum, J. Schweinzer, ASDEX Upgrade Team, Plasma Phys. Control

Fusion 50 (2008) 085009.

[22] R. Fischer, C.J. Fuchs, B. Kurzan, W. Suttrop, E. Wolfrum, ASDEX Upgrade Team,

Fusion Sci. Technol 58, 675 (2010).

[23] W. Suttrop et al., Plasma Phys. Control. Fusion 53 (2011) 124014.

[24] ITER Physics Basis Document, Nucl. Fusion 39, 2175 (1999).

[25] R. M. Mc Dermott et al., Plasma Phys. Control. Fusion 53, 035007 (2011).

[26] V. Mukhovatov et al., Plasma Phys. Control. Fusion 45, A235 (2003).

[27] P.T. Lang et al., J. Nucl. Mater. 290-292, (2001) 374.

[28] G. Tardini, private communication; http://www.ipp.mpg.de/ git/tot/docu.html. MPI für Plasmaphysik Garching, Germany (2011).

[29] G.T.A. Huysmans et al., Plasma Phys. Control. Fusion 51 (2009) 124012.

[30] G. Kocsis et al., "Comparison of the onset of pellet triggered and spontaneous ELMs",

Proc. 37th EPS Conference on CFPP, Dublin (2010), P4.136;

http://ocs.ciemat.es/EPS2010PAP/pdf/P4.136.pdf

[31] L.R. Baylor et al., "ELM pacing by pellet injection on DIII-D and extrapolation to

ITER", Proc. 37th EPS Conference on Controlled Fusion and Plasma Physics, Dublin (2010), P2.117; http://ocs.ciemat.es/EPS2010PAP/pdf/P2.117.pdf 